\title{
84. Formation of the Brain from the Prospective Spinal Cord of Amphibian Embryos*)
}

\author{
By Hirosi TAKAYA \\ Biological Laboratory, Kônan University \\ (Comm. by Yô K. OKADA, м.J.A., June 13, 1955)
}

The central nervous system of amphibian embryos can be divided into three parts: archencephalon, deuterencephalon, and spinal cord. Of these, the first is distinguished from the others not only by certain structural peculiarities, but also by the difference in the surrounding mesodermal tissues. Whereas the deuterencephalon and spinal cord are surrounded ventrally by the notochord and laterally by the muscles or somites, no such mesodermal tissues except loose mesenchyme are found around the archencephalon. This fact, considered together with the fact that the axial mesoderm has the potency to induce neural differentiation, leads us to assume that the mesodermal tissues play an important rôle in the regional differentiation of the central nervous system. However, no evidence has been put forward to verify this assumption. In the following experiments, an attempt was made to ascertain whether the mesoderm can influence the differentiating neural tissue so that the latter changes its regional characters. The experiments were done by means of explanting part of the neural plate. From neurulae at middle or late stages, the brachial part of the neural plate was cut out from about one-third or one-

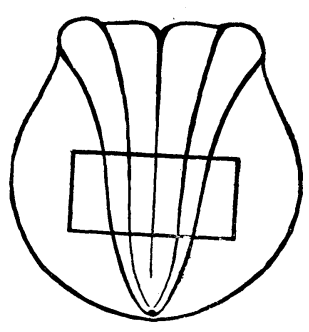

Fig. 1. Neurula of Triturus, showing the position and size of the explanted part of the neural plate

fourth of the whole length (Fig. 1). From this piece of the neural plate the mesodermal lining was wholly or partly removed. The wound was covered by the flank ectoderm, the mesodermal components of which were carefully cleaned off. To compare results, cultivation of the neural plate with the mesodermal lining intact was also carried out. Embryos of Rana japonica, Bufo vulgaris formosus, and Triturus pyrrhogaster were used as materials.

\section{Experimental Results}

When brachial parts of the neural plate were explanted with their mesodermal lining uninjured, differentiation was always in accordance with their prospective fate. In these explants such organs as the * Aided by a grant from the Science Research Fund of the Ministry of Education. 
spinal cord, notochord, somites, and dorsal fin were formed, apparently representing characters of the embryonic trunk. The same neural plate was explanted after removing the entire mesodermal lining. In these explants neural differentiation always occurred. But the neural tissues produced did not form a spinal cord. They generally developed into a large solid mass or a vesicle resembling the forebrain (Fig. 2). In these cases it was marked that no mesodermal tissue was found within the explants. Even loose mesenchyme was very scarce, being produced in only a few specimens. In such cases the neural tissue formed an expanded vesicle with a thin wall (Fig. 3).

Explantation was tested with pieces of the neural plates which were lacking the lateral somite-areas of the lining mesoderm, leaving the central notochordal area uninjured. In all these explants notochord and neural tissues were formed. It was marked that in spite of the operation, most of the explants formed muscle tissues. Especially in the explants of Triturus, muscle differentiation occurred in all the cases examined. Under these conditions, complete failure of muscle differentiation was met with in only a few explants of Rana. When no muscle was formed in the explant, the notochord was well developed, and the neural tissue showed massive proliferation, resulting in the production either of a brain-like vesicle (Fig. 4) or of an irregular solid mass. On the contrary, in those explants in which muscles were produced, neural tissue always developed into a lengthy tube. The walls of the tube were thick where they were in contact with muscle tissues. In consequence, when muscles were found in contact merely with the basal side, the basal wall of the tube alone thickened, thus forming a structure closely resembling the deuterencephalon (Fig. 5). In these cases, ear vesicles were found to be formed adjacent to the neural tube. If muscles were produced in the explants in a large quantity, they generally encircled the neural tissue. In these cases the neural tissue always developed into a narrow tube with a thick wall. Thickening was always on the lateral walls of the tube, resembling the structure of the spinal cord.

Explants of the neural plate lacking the notochordal substrate were made by removing from the mesodermal substrate either the central chordal area alone or the central area together with either side of the lateral muscle-areas. In these explants no formation of the notochord was the rule. But there were a few exceptions in which fragments of the notochord were produced. When the notochord was absent, muscular differentiation was greatly reduced quantitatively and qualitatively. In these explants, however, muscles were found only in small quantity, and very frequently they did not 
show full differentiation. As a rule, these muscles presented no segmentation, and their fibers showed somewhat random arrangement. Exceptionally, in a few specimens of Rana japonica, were found segmental muscles with their fibers regularly arranged. Even in these cases, as shown in Fig. 6, the somites consisted of diffuse fibers, indicating a reduced quantity of muscle tissue. The neural tissue formed together with the somites always became a narrow tube stretched along the longitudinal direction of the somites. From both histological and morphological aspects it was apparent that these tubes represented the typical spinal cord. Also, neural tissues formed

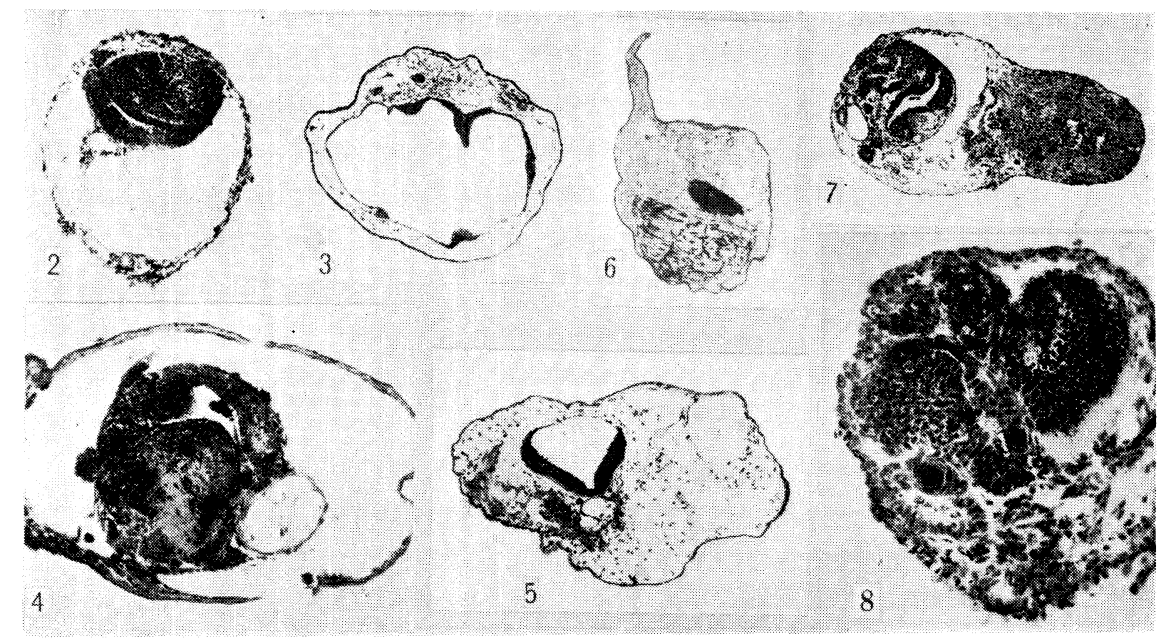

Figs. 2-8. Various types of neural differentiation occurring in explants of the brachial part of the neural plate

Fig. 2. Brain-like mass developed from the neural plate lacking the entire mesodermal substrate (Triturus)

Fig. 3. Expanded vesicle formed in the presence of a small quantity of mesenchymes (Bufo)

Fig. 4. Brain-like mass and notochord developed from explant of the neural plate lacking lateral muscle areas (Rana)

Fig. 5. Deuterencephalon formed together with notochord and muscles (Triturus)

Fig. 6. Somites differentiated in the absence of notochord. Neural tissue forms spinal cord (Rana)

Fig. 7. Neural tissue and muscle mass are separated from one another. Neural tissue develops into a mass, accompanied by ear vesicle (Triturus)

Fig. 8. Eye with lens developed from brachial part of neural plate lacking muscle tissue (Triturus)

together with unsegmented muscles developed into spinal cord so long as they were encircled by the muscles. But if the muscles were situated on one side of the neural tissues, the latter formed hind brain, very frequently accompanied by ear vesicles. There were 
several specimens in which the neural tissues and muscular masses were separated from one another. In such cases proliferation of the neural tissues always occurred to form a large solid mass, such as is shown in Fig. 7.

In the present explants there were many cases in which muscle tissue completely failed to differentiate, and the explants were full of loose mesenchyme. In these cases neural tissue was produced, but it always formed a slender tube with thin walls. In such cases when the mesenchyme was particulary scarce within the explants, the neural tissue formed a solid mass. Although the neural mass produced in these specimens was very irregular in shape, there were two cases in which well differentiated eyes equipped with tapetum and lens were produced in the middle of the neural mass. One of these eyes is reproduced in Fig. 8. From the occurrence of the eye, it is certain that the neural mass, although irregular in shape, represents the archencephalic character. Hence, it may be stated that the neural proliferation which occurs in the absence of mesodermal tissue tends always to the production of the archencephalon.

As above described, all the results of the present experiments demonstrate the importance of the mesodermal substrate for the regional differentiation of the axial nervous system. Of the mesodermal tissues lining the neural plate ectoderm, particular significance is found in the presumptive muscle, because it is disclosed that either brain or spinal cord is produced from the same part of the neural plate ectoderm according as the muscle tissue is absent or present. As a rule, in the absence of mesodermal tissue, neural differentiation resulted in the production of archencephalon, while the neural differentiation in the presence of mesodermal tissue always produced deuterencephalon or spinal cord. In this latter case, the quantity of the muscles bears significance: the more muscle tissue is produced, the more caudal is shifted the character of the neural organs produced.

\section{Discussion}

The present experiments have disclosed that the neural plate ectoderm can differentiate into neural tissue even when the lining mesoderm is thoroughly removed. From this fact, it naturally follows that a passing contact of the mesoderm is sufficient to induce neuralization of the ectoderm. However, the neural tissue induced shows regional characteristics depending on the simultaneous differentiation of the accompanying mesodermal tissues. Whereas, in the absence of mesodermal tissue, neural differentiation was always towards the production of archencephalon, in the presence of meso- 
dermal tissue, especially of muscles and somites, deuterencephalon and spinal cord always developed.

From these facts of our experiments, we are inclined to assume that in the development of the central nervous system, at least, two steps can be distinguished. The first step consists merely in an evocation of the ectoderm, in which neuralization takes place through the influence of the lining mesoderm. In this step it must be concluded that no factor is involved to determine the regional character of the neuralizing ectoderm, since our experiments revealed that the brachial part of the neural plate, when it was cultivated after removing the entire mesodermal substrate, always developed into neural vesicles or solid masses showing an archencephalic character. Since this part of the neural plate, normally, in its natural position, develops into the spinal cord, such formation of the archencephalon clearly manifests that the evocated ectoderm is still in a labile state so that its differentiating tendency is altered according to the way in which the condition is changed.

In the neuralizing tissue, determination of the regional character may take place in the second step. The factors operating in this step may be of two kinds: one includes the internal factors involved in the neuralizing tissue itself, and the other, the external factors issuing from the surrounding mesodermal tissues. As found in our experiments, the neural differentiation which occurred in the absence of mesoderm, always consisted of large vesicles or solid masses of neural tissues which show an archencephalic character, and no other structures were formed. From this fact, we may be permitted to assume that there exists in the differentiating neural tissue a definite tendency probably brought about by a factor or factors immanent in the tissue itself. A similar idea which assumes an intrinsic differentiating tendency of the neural tissue has already been advanced by Nieuwkoop ('52).

The intrinsic differentiating tendency of the neural tissue may be modified by external factors issuing from the surrounding mesodermal tissues. Of the mesodermal tissues, mesenchyme, and muscle seem to exert different influences, and differentiating muscle tissues have been found in our experiments to have the most conspicuous effect. Through the influence of the muscle tissues, the intrinsic tendency of the differentiating neural tissue is modified so that the neural tissue comes to form organs more caudal than the archencephalon. As described above, the formation of a lengthy tube was the rule when neural tissue was developed together with the mesodermal tissues. Neural tubes were slender with thin walls when they were encircled merely by loose mesenchyme. When the muscles 
were formed on one side of the tube, that side of the tube always thickened to form a deuterencephalon. When the tube was flanked bilaterally by muscles or somites, thickening of the lateral walls occurred with the resulting production of a typical spinal cord.

In so far as our present experiments are concerned, no direct influence of the notochord was found, at least upon the regional differentiation of the neural plate. Indirectly, however, the notochord may bear significance. Evidences indicating a secondary significance of the notochord have been gathered from the present experiments, and their description will be given in our next publication.

\section{References}

Holtfreter, J.,: Boux' Arch., 127 (1933).

Nieuwkoop, P. D.,: J. Exp. Zool., 120 (1952).

Takaya, H.,: Zool. Mag., 63 (1953).

Waddington, C. H.,: J. Exp. Biol., 29 (1952).

Waddington, C. H., and Deucher, E. M.,: Ibid., 29 (1952).

Woellwarth, C. von,: Roux' Arch., 145 (1952). 